\title{
Beneficial effects of dexmedetomidine on early postoperative cognitive dysfunction in pediatric patients with tonsillectomy
}

\author{
CHUANLAI HAN ${ }^{1,2}$, RONG FU ${ }^{2}$ and WEIFU LEI ${ }^{1}$ \\ ${ }^{1}$ Department of Anesthesiology, Qilu Hospital of Shandong University, Jinan, Shandong 250012; \\ ${ }^{2}$ Department of Anesthesiology, Nanjing Traditional Chinese Medical Hospital, Nanjing, Jiangsu 210001, P.R. China
}

Received August 8, 2016; Accepted February 8, 2018

DOI: $10.3892 /$ etm.2018.6180

\begin{abstract}
According to clinical investigations, early postoperative cognitive dysfunction is the most common adverse event in pediatric patients after tonsillectomy. A previous study has indicated that dexmedetomidine (DEX) is an efficient drug for the treatment of postoperative cognitive dysfunction. However, the efficacy of DEX in alleviating early postoperative cognitive dysfunction in pediatric patients following tonsillectomy has remained elusive, which was therefore assessed in the present study. A total of 186 children presenting with cognitive dysfunction subsequent to tonsillectomy were recruited to analyze the efficacy of DEX. Patients were randomly divided into two groups and received intravenous treatment with DEX $(n=112)$ or placebo $(n=74)$. Duration of treatment, dose-limiting toxicities (DLT) and maximum tolerated dose (MTD) of DEX were evaluated in a preliminary experiment. The improvement of postoperative cognitive function in children with tonsillectomy was analyzed with a Mini-Mental State Examination (MMSE) following treatment with DEX. A 40-item quality of life (MONEX-40) questionnaire was used to assess the efficacy of DEX. The plasma levels of interleukin (IL)-6, IL-1, tumor necrosis factor (TNF)- $\alpha$, superoxide dismutase (SOD), neuron-specific enolase (NSE), $\mathrm{C}$-reactive protein (CRP), cortisol and melatonin were also analyzed. The preliminary experiment determined that the DLT was $10 \mathrm{mg} / \mathrm{kg}$ and the MTD was $15 \mathrm{mg} / \mathrm{kg}$. In the major clinical trial, it was revealed that MMSE scores in the DEX treatment group were markedly improved, indicating that DEX had a beneficial effect in pediatric patients with early postoperative cognitive dysfunction after tonsillectomy. In addition, IL-1and TNF- $\alpha$ were downregulated, while IL-6 and SOD were upregulated in patients with cognitive dysfunction after treatment with DEX compared with those in the placebo
\end{abstract}

Correspondence to: Professor Weifu Lei, Department of Anesthesiology, Qilu Hospital of Shandong University, 107 Wenhua West Road, Jinan, Shandong 250012, P.R. China

E-mail: leiweifuchild@163.com

Key words: dexmedetomidine, postoperative cognitive dysfunction, tonsillectomy, inflammation group. Furthermore, DEX treatment markedly decreased the serum levels of CRP, NSE cortisol and melatonin, which are associated with the occurrence of postoperative cognitive dysfunction in pediatric patients following tonsillectomy. In conclusion, intravenous administration of DEX at a dose of $10 \mathrm{mg} / \mathrm{kg}$ improves postoperative cognitive function in pediatric patients with tonsillectomy by decreasing the serum levels of inflammatory factors and stress-associated signaling molecules. Trial registration no. QLSDHOS0200810102C (Qilu Hospital of Shandong University, Jinan, China).

\section{Introduction}

Children undergoing tonsillectomy are at risk of experiencing complications, including postoperative haemorrhage, nausea and vomiting, and cognitive dysfunction (1). A previous study has identified the clinical features of children undergoing tonsillectomy and presented the adverse outcomes and therapeutic schedule (2). Postoperative vomiting and pain due to tonsillectomy are two of the most frequent complications in pediatric patients $(3,4)$. In addition, Eisert et al (5) indicated that bleeding remains the most important complication of tonsillectomy in pediatric patients and coagulation tests are widely applied to assess bleeding events. Furthermore, the immunological sequela of tonsillectomy in pediatric patients has also been indicated by physicians (6). Of note, cognitive dysfunction following tonsillectomy is a post-operative complication that occurs most frequently in pediatric patients $(7,8)$. Therefore, prevention and treatment of postoperative cognitive dysfunction following tonsillectomy is essential for pediatric patients (9).

Cognitive impairment following tonsillectomy is a serious clinical problem as it reduces intelligence and emotional stability $(10,11)$. Acute coagulatory dysfunction during adenoidectomy and tonsillectomy has been investigated in a previous case report on a pediatric patient (12). In addition, postoperative cognitive dysfunction, characterized by impaired consciousness and disordered thinking patterns, represents a major complication in pediatric patients after anesthesia and tonsillectomy (13). Although tonsillectomy provides numerous advantages, including less bleeding and fewer infections compared with conventional open procedures, cognitive dysfunction occurs due to adverse effects on cerebral function $(14,15)$. Previous evidence indicates that cerebral oxygenation is decreased following tonsillectomy, as suggested by lightheadedness, nightmares, 
nausea, vomiting and constipation, which may be correlated with neurocognitive changes in pediatric patients (16). These results suggest that the cognitive competence of the patients, which is regulated by the nerve center within the brainstem, may be affected by tonsillectomy in pediatric patients.

Dexmedetomidine (DEX) is a highly selective $\alpha 2$-adrenergic receptor agonist and acts as a multifunctional drug in the treatment of various human diseases (17). A previous study has suggested that DEX is efficient in the treatment of nerve diseases through the beneficial effects of acting as an anxiolytic, sedative, analgesic and blocking the sympathetic nervous system (18). In addition, a clinical study has indicated that DEX has analgesic, anxiolytic and anti-delirium effects, while causing little respiratory depression (19). DEX treatment may improve behavioral disturbances, including aggression, agitation and cognitive impairment (20). These neurological function impairments may occur in pediatric patients with postoperative cognitive dysfunction. Therefore, it was hypothesized that DEX may be beneficial for restoring cognitive function in pediatric patients following tonsillectomy.

In the present study, the effects of DEX administration were assessed in pediatric patients with cognitive impairment after tonsillectomy. Although a previous study has suggested that DEX is recommended for decreasing the risk of postoperative vomiting, as well as to alleviate pain, inflammation and nausea for patients in intensive care (21), the therapeutic efficacy of DEX on the restoration of cognitive function has remained elusive. In addition to the influence of DEX on the recovery of cognitive impairment, its effects on the levels of interleukin (IL)-6 and -1, tumor necrosis factor (TNF)- $\alpha, \mathrm{C}$-reactive protein (CRP), neuron-specific enolase (NSE), superoxide dismutase (SOD), cortisol and melatonin in pediatric patients following tonsillectomy were also assessed. The results indicate that DEX improves cognitive impairment in pediatric patients following tonsillectomy, at least in part, through the regulation of IL-6, CRP, cortisol and melatonin levels.

\section{Materials and methods}

Patients. Pediatric patients aged 6-12 years who had undergone tonsillectomy at Qilu Hospital of Shandong University (Jinan, China) between May 2014 and July 2015 were subjected to a Mini Mental State Examination (MMSE) and requested to complete a 40-item quality of life (MONEX-40) questionnaire. The MMSE was used to screen for cognitive dysfunction. Only patients post tonsillectomy with post-operative cognitive dysfunction were included in the study. The patients were randomly divided into two groups, and double-blinded trails were performed. Further details, including a description of the inclusion/exclusion criteria and the allocation method, are specified in a previously published study (22). In a preliminary experiment, the patients with cognitive dysfunction received DEX (1.0, 5.0, 10.0, 15.0 and $20.0 \mathrm{mg} / \mathrm{kg} /$ day) or placebo (PBS) through intravenous injection for 4 weeks. The dose-limiting toxicities (DLT) and maximum tolerated dose (MTD) of DEX were 10 and $15 \mathrm{mg} / \mathrm{kg}$, respectively, determined by the common treatment-emergent adverse events of DEX as described previously (23).

Study design. The double-blinded study was performed in 3 phases: Baseline stage, double-blinded treatment phase (4-week dose-titration treatment, preliminary experiment) and 4-week post-treatment (maintenance treatment) of those patients who volunteered to continue to complete the ongoing extension study. The patients were randomized into two groups which were treated once a day with DEX or placebo in a double-blinded manner. In the final investigation, treatment was continued with the $10 \mathrm{mg} / \mathrm{kg}$ dose of DEX or placebo to achieve the ideal effect throughout the maintenance period.

Outcome measures. The MMSE (24) and The 40-item Monell Extended Sniffin' Sticks Identification Test (MONEX-40) (40 items for assessing functional limitation) (25) were used for assessing the pediatric patients with cognitive dysfunction at prior to treatment and post-treatment. Clinical cognitive function scores were evaluated as described in a previous study (26).

Efficacy and safety assessments. Efficacy assessments, including the median percent reduction scores and response rate, were analyzed with the baseline values as a reference during the 4-week double-blinded treatment period with DEX $(10 \mathrm{mg} / \mathrm{kg})$ or placebo. In addition, overall safety and pharmacokinetic analyses were performed according to the protocols of previous clinical studies $(27,28)$. The safety assessments regarding the most frequent treatment-emergent adverse events were performed in all randomized patients. The dosage of DEX was determined by DLT as described above. Dose-response analysis was performed when the last dose of the drug was injected as described previously (29).

ELISA. In the present study, the serum levels of IL-6 (cat. no. D6050), IL-1 (cat. no. DLB50), TNF- $\alpha$ (cat. no. DTA00C), SOD (cat. no. DYC3419-2), NSE (cat. no. DY5169-05), CRP (cat. no. DCRP00), cortisol (cat. no. KGE008B) (all Bio-Rad Laboratories, Inc., Hercules, CA, USA) and melatonin (cat. no. KA1166; Abnova, Taipei, Taiwan) were assessed using commercialized ELISA kits. The ELISAs were performed according to the manufacturer's protocols. The results were determined by measuring the absorbance at $450 \mathrm{~nm}$ with an ELISA reader and finally converted to the concentrations of IL-6, IL-1, TNF- $\alpha$, SOD, NSE, CRP, cortisol and melatonin.

Statistical analysis. All values are expressed as the mean \pm standard error of the mean. All data were analyzed using SPSS software version 19.0 (IBM Corp., Armonk, NY, USA). Statistical significance of differences between mean values were assessed by Student's t-test for paired data. Comparisons of data between multiple groups were performed by one-way analysis of variance followed by Tukey's post hoc test. Responder rates and treatment-emergent adverse events were analyzed with the $\chi^{2}$ test. $\mathrm{P}<0.05$ was considered to indicate a statistically significant difference.

\section{Results}

Patient characteristics. A total of 186 pediatric patients with early postoperative cognitive dysfunction following tonsillectomy were recruited for the present study. The characteristics of the patients are summarized in Table I. The gender distribution within the cohort was equal. The cognitive dysfunction 
Table I. Characteristics of the study population post tonsillectomy.

\begin{tabular}{lc}
\hline Characteristic & Value \\
\hline Total patients with post-operative cognitive & $186(100)$ \\
dysfunction & \\
Gender & \\
Male & $90(48)$ \\
Female & $96(52)$ \\
Median age, years (range) & $8.5(6-12)$ \\
MMSE & $16.5 \pm 3.2$ \\
MONEX-40 & $134.4 \pm 12.5$ \\
Pain scores & $7.2 \pm 2.4$ \\
Drug therapy & $186(100)$ \\
DEX & $112(60)$ \\
Placebo & $74(40)$ \\
\hline
\end{tabular}

Values are expressed as $\mathrm{n}(\%)$ or as the mean \pm standard error of the mean unless otherwise specified. MMSE, Mini Mental State Examination; MONEX-40, 40-item quality of life questionnaire; DEX, dexmedetomidine.

of the patients was determined by the MMSE at prior to treatment and post treatment. No other medications or painkillers were taken during the treatment period.

Duration of treatment, DLT and MTD of DEX. The median overall duration of DEX treatment was 4 weeks followed by maintenance treatment. The dosing cohorts of DEX were 1.0, $5.0,10.0,15.0$ and $20 \mathrm{mg} / \mathrm{kg}$ to evaluate the optimal dosage. According to the results in Table II, the MTD of DEX was $20 \mathrm{mg} / \mathrm{kg}$ once a day. The DLT was determined as $15 \mathrm{mg} / \mathrm{kg}$ of DEX once a day. The group treated with the lowest dose of DEX presented with the fewest side effects. Analysis indicated that the common treatment-emergent adverse events of DEX were hypertension, fatigue, proteinuria, hypertriglyceridemia, constipation and peripheral edema. Of note, most of the patients treated with the MTD of DEX required a dose reduction due to cumulative toxicity. Therefore, most of the patients that were subsequently enrolled received a dose of $10.0 \mathrm{mg} / \mathrm{kg}$ DEX to achieve ideal tolerability and therapeutic efficacy in the pediatric patients with early postoperative cognitive dysfunction.

Treatment-emergent adverse events associated with $D E X$. Pediatric patients with early postoperative cognitive dysfunction after tonsillectomy received DEX therapy with post-baseline safety evaluation were included in the safety population. After the administration of the last dose of DEX, it was determined that the most common treatment-emergent adverse events of DEX (10 mg/kg) were hypertension and proteinuria ( $\geq 10 \%$ each) (Table III). The administration of $\mathrm{DEX}$ at $\geq 15 \mathrm{mg} / \mathrm{kg}$ is not advisable due to more side effects and therefore, few patients were treated with such doses. Of the total patient population, 96 completed the overall maintenance period of the phase III study.
Analysis of the efficacy of DEX in alleviating cognitive impairment in pediatric patients after tonsillectomy. In order to investigate the efficacy of DEX in improving of cognitive function in children affected after tonsillectomy, the cognitive competence was assessed in 186 patients using the MMSE and MONEX-40. As presented in Fig. 1, it was demonstrated that DEX treatment significantly improved the cognitive competence of children with cognitive dysfunction determined by the MMSE scores. In addition, assessment with the MONEX-40 questionnaire indicated that DEX treatment reduced postoperative complications (daytime sedation, lightheadedness, nightmares, nausea, vomiting and constipation) compared with the placebo group (Fig. 2). It was also observed that the plasma concentration of NSE was increased in patients after DEX treatment compared with that in the placebo group (Fig. 3). Of note, the plasma concentration of CRP was decreased in the DEX group (Fig. 4). Furthermore, the serum levels of melatonin were increased in patients after DEX treatment compared with those in the placebo group (Fig. 5). Importantly, the plasma concentration of cortisol was upregulated by DEX compared with that in the placebo group (Fig. 6). For all these factors no significant changes were observed in the placebo group between the prior to treatment and post treatment time points. These results suggest that DEX improves cognitive competence through the regulation of nerve growth factors in pediatric patients with cognitive dysfunction after tonsillectomy.

Analysis of inflammatory factors in pediatric patients with tonsillectomy after treatment with DEX. The inflammatory factors in pediatric patients with post-tonsillectomy cognitive dysfunction after treatment with DEX were analyzed. It was indicated that the plasma levels of IL-1 were increased after DEX treatment (Fig. 7). In addition, DEX treatment downregulated the plasma levels of TNF- $\alpha$ to inhibit the inflammatory response (Fig. 8). Furthermore, the plasma concentration of IL-6 was increased after DEX treatment (Fig. 9). Treatment with DEX also improved SOD levels in the plasma, which may contribute to the recovery of cognitive dysfunction after tonsillectomy (Fig. 10). These observations indicate that inflammatory factors in pediatric patients with tonsillectomy were improved after treatment with DEX. For the above factors no significant changes were observed in the placebo group between the prior to treatment and post treatment time points.

\section{Discussion}

Previous studies have indicated that early postoperative cognitive dysfunction is one of the most common adverse effects in pediatric patients after tonsillectomy $(30,31)$. DEX has been approved by the Chinese Food and Drug Administration for the treatment of inflammation (32). In addition, it has been suggested that DEX is an efficient drug for the treatment of postoperative cognitive dysfunction $(33,34)$. In the present study, the clinical therapeutic effects of DEX on recovery of cognitive dysfunction in pediatric patients that had undergone tonsillectomy were investigated. In a preliminary study, the DLT and MTD of DEX were evaluated in pediatric patients with cognitive dysfunction after tonsillectomy. The 
Table II. Overall incidence of treatment-emergent adverse events of DEX.

\begin{tabular}{lcccc}
\hline & & \multicolumn{3}{c}{ DEX $(\mathrm{mg} / \mathrm{kg})$} \\
\cline { 3 - 5 } Adverse event & Total $(\mathrm{n}=32)$ & $1-10(\mathrm{n}=10)$ & $15(\mathrm{n}=14)$ & $20(\mathrm{n}=8)$ \\
\hline Hypertension & 5 & 1 & 2 & 2 \\
Fatigue & 3 & 1 & 1 & 1 \\
Proteinuria & 4 & 0 & 1 & 3 \\
Hypertriglyceridemia & 2 & 0 & 1 & 2 \\
Constipation & 3 & 1 & 1 & 1 \\
Edema peripheral & 3 & 0 & 1 & 2 \\
\hline
\end{tabular}

DEX, dexmedetomidine.

Table III. Severity of treatment-emergent hypertension and proteinuria as adverse events of DEX.

\section{Adverse}

event/grade

Hypertension

$$
1
$$

2

3

Proteinuria

1

2

3
Hypertension$$
\text { (1) }
$$

2

(1)
3
$\operatorname{DEX}(\mathrm{mg} / \mathrm{kg})$

\begin{tabular}{ccc}
\hline $1-10(\mathrm{n}=10)$ & $15(\mathrm{n}=14)$ & $20(\mathrm{n}=8)$ \\
1 & 2 & 2 \\
0 & 0 & 1 \\
0 & 1 & 1 \\
1 & 1 & 0 \\
0 & 1 & 3 \\
0 & 0 & 1 \\
0 & 1 & 1 \\
0 & 0 & 1
\end{tabular}

DEX, dexmedetomidine. Grading scale of hypertension and proteinuria was determined by WHO hypertension and proteinuria grade.

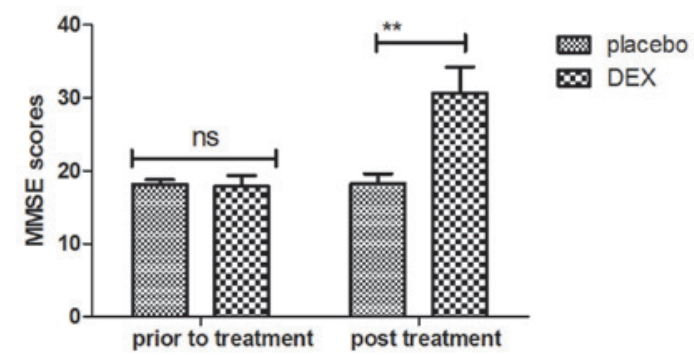

Figure 1. Evaluation the effects of DEX on cognitive dysfunction using MMSE scores. ${ }^{* *} \mathrm{P}<0.01$ vs. control. MMSE, Mini Mental State Examination; DEX, dexmedetomidine; ns, not significant.

treatment-emergent adverse events of DEX were analyzed to evaluate the clinical efficacy and pharmacodynamics. Furthermore, the serum levels of inflammatory and nerve growth factors were analyzed to determine the mechanism of DEX-induced recovery of cognitive function. The results indicate that DEX improves cognitive dysfunction in children after tonsillectomy through inhibition of the expression of inflammatory factors and enhancement of neuroprotective protein expression. These results suggest that DEX may be an efficient cognitive function-enhancing drug for the treatment

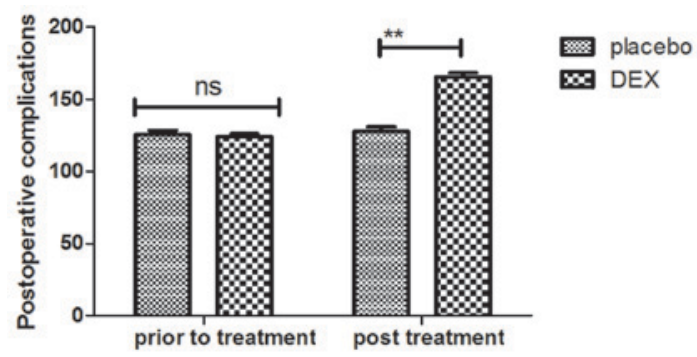

Figure 2. Analysis of efficacy of DEX on improvement of postoperative complications in children with tonsillectomy. ${ }^{* *} \mathrm{P}<0.01$ vs. control. DEX, dexmedetomidine; ns, not significant.

of tonsillectomy-associated cognitive impairment in pediatric patients.

Postoperative cognitive dysfunction is a multifactorial adverse event and most frequently occurs in pediatric and elderly patients after surgery (35). Surgical therapy is the most common clinical treatment of frequent tonsil inflammation. Tonsillectomy is also one of the most common surgical procedure performed in preschool children (36). Although tonsillectomy provides numerous benefits, various adverse reactions, including throat pain, haemorrhage, tonsillar fossa 


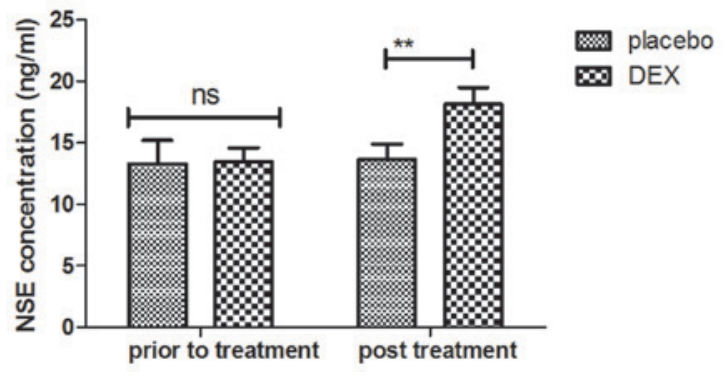

Figure 3. Analysis of efficacy of DEX on NSE plasma concentration after an 8 -week treatment period. ${ }^{* *} \mathrm{P}<0.01$ vs. control. DEX, dexmedetomidine; NSE, neuron-specific enolase; ns, not significant.

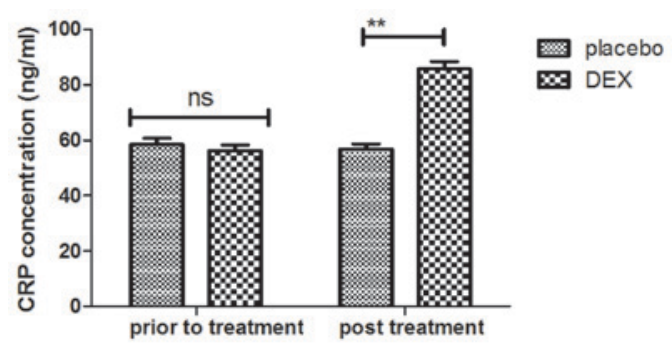

Figure 4. Detection of CRP plasma concentration levels between DEX and placebo groups. ${ }^{* *} \mathrm{P}<0.01$ vs. control. DEX, dexmedetomidine; CRP, C-reactive protein; ns, not significant.

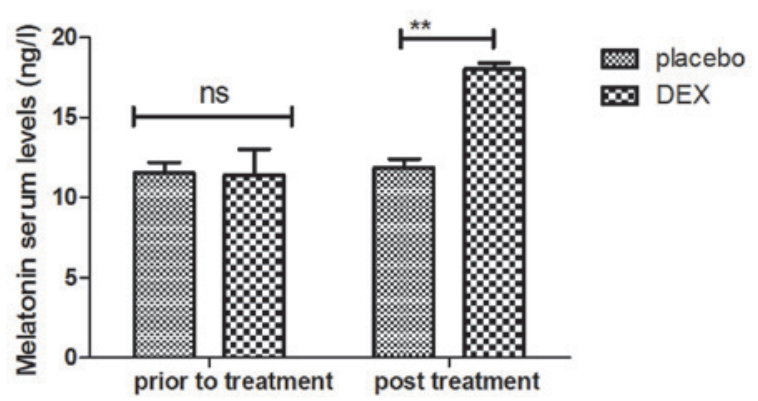

Figure 5. Changes of melatonin serum levels in pediatric patients with post-operative cognitive dysfunction after DEX treatment. ${ }^{* *} \mathrm{P}<0.01$ vs control. DEX, dexmedetomidine; ns, not significant.

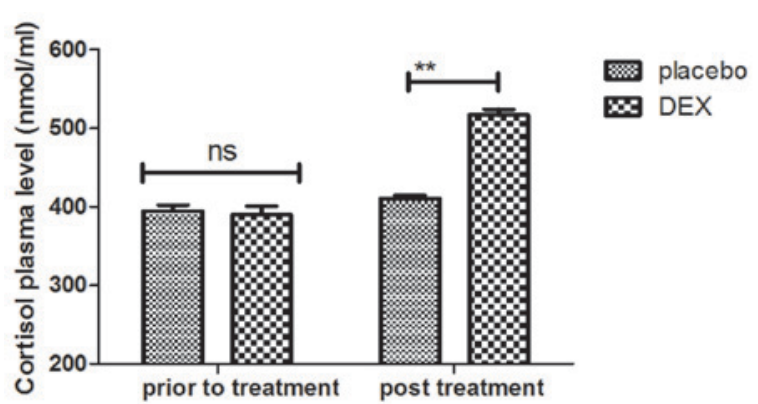

Figure 6. Analysis of efficacy of DEX in regulating the cortisol plasma concentration. ${ }^{* *} \mathrm{P}<0.01$ vs. control. DEX, dexmedetomidine; ns, not significant.

epithelisation and even cognitive dysfunction, may occur in patients after tonsillectomy (37). A previous study has reported that cognitive dysfunction may affect children for up to 6 months after the operation (13). Therefore, the treatment

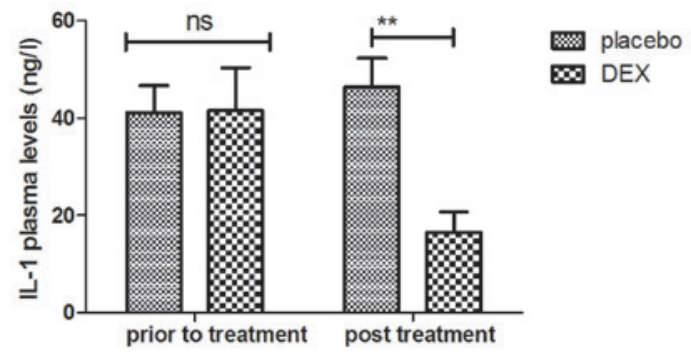

Figure 7. Clinical analysis of IL-6 plasma levels after DEX treatment. * $\mathrm{P}<0.01$ vs. control. DEX, dexmedetomidine; IL, interleukin; ns, not significant.

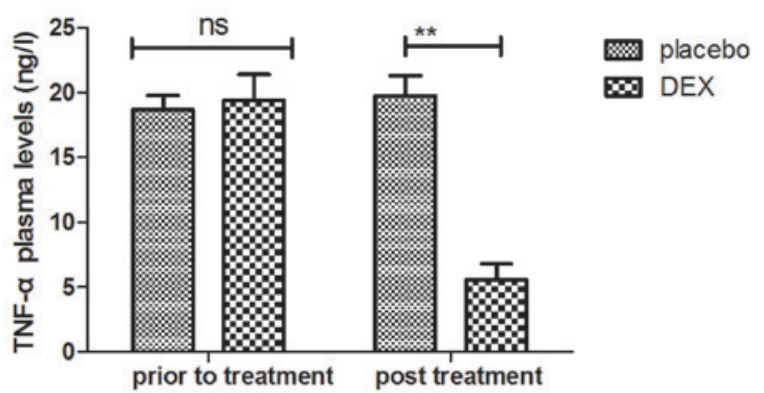

Figure 8. Clinical analysis of IL-1 plasma levels after DEX treatment. ${ }^{* *} \mathrm{P}<0.01$ vs. control. DEX, dexmedetomidine; IL, interleukin; ns, not significant.

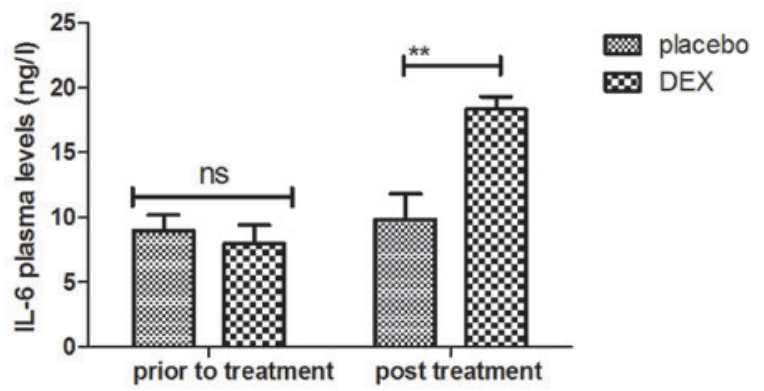

Figure 9. Analysis of the effect of DEX on the plasma concentration of TNF- $\alpha .{ }^{* * *} \mathrm{P}<0.01$ vs. control. DEX, dexmedetomidine; TNF, tumor necrosis factor; ns, not significant.

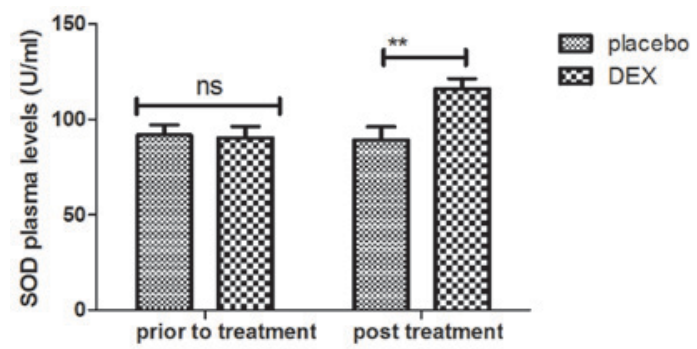

Figure 10. Analysis of the effect of DEX on the plasma concentration of SOD. ${ }^{* *} \mathrm{P}<0.01$ vs. control. DEX, dexmedetomidine; SOD, superoxide dismutase; ns, not significant.

of postoperative cognitive dysfunction is essential for pediatric patients in the clinic.

DEX has been increasingly applied during clinical surgery as a regional anesthetic (38). It is a multifunctional drug and a highly efficient and specific $\alpha 2$ agonist, which may reduce 
the release of norepinephrine, the activity of norepinephrine receptors exert anxiolytic and calming effects, and ameliorate sleep cycle disorders $(22,39)$. Numerous clinical trials and studies have demonstrated that DEX protects cells in the hippocampus against injury and reduces ischemia-reperfusion injury of human organs $(40,41)$. The present study further explored the neuroprotective effects of DEX in pediatric patients with cognitive dysfunction after tonsillectomy. DEX has sedative, analgesic and hypnotic effects, and antagonizes sympathetic activity, which results in improvement of cognitive competence. A previous study has indicated that recovery from cognitive dysfunction required $>6$ weeks for pediatric patients with tonsillectomy (42). The present study only implemented a 4-week observation period and in the placebo group, no improvement was seen; however, it should be clarified that the post-operative cognitive dysfunction following tonsillectomy is a non-permanent condition, and that recovery may take $>4$ weeks. However, further study is required to assess the average time of recovery from cognitive dysfunction in pediatric patients following tonsillectomy procedures, and the specific effects of DEX and the underlying molecular mechanisms of its cerebral protection require to be elucidated.

An integrative review has examined the use of DEX as an anesthetic for monitored anesthesia care and regional anesthesia, and has evaluated the effect of DEX on the incidence of postoperative cognitive dysfunction after non-cardiac and non-neurologic surgery (43). DEX acts as an antagonist of transmembrane $\mathrm{G}$ protein-coupled receptor that significantly contributes to distribution of hippocampal focal adhesion kinase tyrosine phosphorylation in the peripheral nervous system, autonomic ganglia and central nervous system (44). The major function of DEX is to selectivity activate $\alpha 2$ adrenergic receptors to regulate the locus coeruleus of the central nervous system. A phase III double-blinded, randomized controlled trial has suggested that the effects of a single low dose of dexamethasone prior to non-cardiac and non-neurologic surgery and general anesthesia provides beneficial effects on postoperative cognitive dysfunction (45). Another clinical trial reported on the preventive effects of low-dose DEX on postoperative cognitive dysfunction and the quality of recovery in oral cancer patients through modulating the kinetics of cortisol, expression of inflammatory cytokines and plasma concentration of melatonin (46). The present study indicates that DEX has a beneficial effect on the recovery from cognitive dysfunction in pediatric patients after tonsillectomy through inhibition of the expression of inflammatory factors and enhancement of neuroprotective protein expression. However, as it was not determined whether the patients had any cognitive dysfunction prior to surgery, it may not have been surgery-associated in all cases.

In conclusion, the present study observed that DEX increased the plasma concentration of cortisol in pediatric patients with early postoperative cognitive dysfunction after tonsillectomy, and cortisol is negatively associated with the risk of cognitive dysfunction. Of note, DEX reduced the serum levels of IL-1 and TNF- $\alpha$ to decrease nerve injury. Furthermore, DEX treatment improved the cognitive ability and relieved cognitive dysfunction caused by tonsillectomy. Taken together, DEX improves cognitive impairment in children after tonsillectomy through regulation of the expression of inflammatory factors and neuroprotective proteins, which may widely apply for impairments of brain function caused by tonsillectomy.

\section{Acknowledgements}

Not applicable.

\section{Funding}

No funding was received.

\section{Availability of data and materials}

The datasets used and/or analyzed during the current study are available from the corresponding author on reasonable request.

\section{Authors' contributions}

WL designed the study. $\mathrm{CH}$ and RFperformed the experiments and analyzed the data.

\section{Ethical approval and consent to participate}

This phase-III study (no. QLSDHOS0200810102C) was performed in strict accordance with the recommendations in the Guidelines of Qilu Hospital of Shandong University (Jinan, China) between October 2008 and May 2014. All patients and their guardians were required to review trial protocols and their amendments, and provide informed consent.

\section{Consent for publication}

All patients provide written informed consent for the publication of their data.

\section{Competing interests}

The authors declare that they have no competing interests.

\section{References}

1. Elgueta MF, Echevarría GC, De la Fuente N, Cabrera F, Valderrama A, Cabezón R, Muñoz HR and Cortinez LI: Effect of intravenous fluid therapy on postoperative vomiting in children undergoing tonsillectomy. Br J Anaesth 110: 607-614, 2013.

2. Buckley A and Savage E: Preoperative information needs of children undergoing tonsillectomy. J Clin Nurs 19: 2879-2887, 2010.

3. Leong AC: A randomised controlled trial to compare postoperative pain in children undergoing tonsillectomy using cold steel dissection with bipolar haemostasis versus coblation technique. Clin Otolaryngol 34: 579-580, 2009.

4. Parker D, Howe L, Unsworth V and Hilliam R: A randomised controlled trial to compare postoperative pain in children undergoing tonsillectomy using cold steel dissection with bipolar haemostasis versus coblation technique. Clin Otolaryngol 34: 225-231, 2009.

5. Eisert S, Hovermann M, Bier H and Göbel U: Preoperative screening for coagulation disorders in children undergoing adenoidectomy (AT) and tonsillectomy (TE): Does it prevent bleeding complications? Klin Padiatr 218: 334-339, 2006.

6. Reichel O, Mayr D, Winterhoff J, de la Chaux R, Hagedorn H and Berghaus A: Tonsillotomy or tonsillectomy?-a prospective study comparing histological and immunological findings in recurrent tonsillitis and tonsillar hyperplasia. Eur Arch Otorhinolaryngol 264: 277-284, 2007. 
7. Prows CA, Zhang $X$, Huth MM, Zhang K, Saldaña SN, Daraiseh NM, Esslinger HR, Freeman E, Greinwald JH, Martin LJ and Sadhasivam S: Codeine-related adverse drug reactions in children following tonsillectomy: A prospective study. Laryngoscope 124: 1242-1250, 2014.

8. Merry AF, Edwards KE, Ahmad Z, Barber C, Mahadevan M and Frampton C: Randomized comparison between the combination of acetaminophen and ibuprofen and each constituent alone for analgesia following tonsillectomy in children. Can J Anaesth 60: 1180-1189, 2013.

9. Liasis A, Nischal KK, Leighton S, Yap S, Hayward R and Dunaway D: Adenoid-tonsillectomy to treat visual dysfunction in a child with craniosynostosis. Pediatr Neurosurg 41: 197-200, 2005.

10. Maini S, Osborne JE, Fadl HM, Spyridakou C, Ogunyemi L and Hill P: Temporomandibular joint dysfunction following tonsillectomy. Clin Otolaryngol Allied Sci 27: 57-60, 2002

11. Garnett JD and Ramadan HH: Swallowing dysfunction after tonsillectomy. Otolaryngol Head Neck Surg 114: 813-817, 1996.

12. Chu JT, Wu WM and Liu FF: Acute coagulatory dysfunction during adenoidectomy and tonsillectomy in a child Zhonghua Er Bi Yan Hou Tou Jing Wai Ke Za Zhi 43: 57, 2008 (In Chinese)

13. Hart JA and Glickman-Simon R: Phytomedicines for Helicobacter pylori, garlic for hypertension, intraoperative acupuncture for tonsillectomy, Omega-3 fatty acids for cognitive decline, qigong for COPD. Explore (NY) 12: 141-145, 2016

14. Jimenez N, Anderson GD, Shen DD, Nielsen SS, Farin FM, Seidel K and Lynn AM: Is ethnicity associated with morphine's side effects in children? Morphine pharmacokinetics, analgesic response, and side effects in children having tonsillectomy. Paediat Anaesth 22: 669-675, 2012.

15. Bellis JR, Pirmohamed M, Nunn AJ, Loke YK, De S, Golder S and Kirkham JJ: Dexamethasone and haemorrhage risk in paediatric tonsillectomy: A systematic review and meta-analysis. Br J Anaesth 113: 23-42, 2014

16. Sutters KA, Miaskowski C, Holdridge-Zeuner D, Waite S, Paul SM, Savedra MC and Lanier B: Time-contingent dosing of an opioid analgesic after tonsillectomy does not increase moderate-to-severe side effects in children. Pain Management Nurs 6: 49-57, 2005 .

17. Mason KP and Lerman J: Review article: Dexmedetomidine in children: Current knowledge and future applications. Anesth Analg 113: 1129-1142, 2011.

18. Wang X, Zhao B and Li X: Dexmedetomidine attenuates isoflurane-induced cognitive impairment through antioxidant, anti-inflammatory and anti-apoptosis in aging rat. Int J Clin Exp Med 8: 17281-17288, 2015.

19. Ohtsuka M: Dexmedetomidine for postoperative sedation in elderly patients with cognitive impairment. Masui 61: 379-383, 2012 (In Japanese).

20. Prommer E: Review article: Dexmedetomidine: Does it have potential in palliative medicine? Am J Hosp Palliat Care 28 276-283, 2011

21. Tejani AM and Schwenger E: Comment on dexmedetomidine systematic review and meta-analysis methodology. Inten Care Med 36: 1974-1975, 2010.

22. David MD and De Marchi L: Dexmedetomidine sedation for awake tracheotomy: Case report and literature review. J Clin Anesth 22: 360-362, 2010.

23. Penel N, Adenis A, Clisant S and Bonneterre J: Nature and subjectivity of dose-limiting toxicities in contemporary phase 1 trials: Comparison of cytotoxic versus non-cytotoxic drugs. Invest New Drugs 29: 1414-1419, 2011.

24. Ong HL, Subramaniam M, Abdin E, Wang P, Vaingankar JA, Lee SP, Shafie S, Seow E and Chong SA: Performance of Mini-Mental State Examination (MMSE) in long-stay patients with schizophrenia or schizoaffective disorders in a psychiatric institute. Psychiat Res 241: 256-262, 2016.

25. Freiherr J, Gordon AR, Alden EC, Ponting AL, Hernandez MF, Boesveldt S and Lundström JN: The 40-item Monell Extended Sniffin' sticks identification test (MONEX-40). J Neurosci Methods 205: 10-16, 2012.

26. Ansari NN, Naghdi S, Hasson S, Valizadeh L and Jalaie S: Validation of a Mini-Mental State Examination (MMSE) for the Persian population: A pilot study. Appl Neuropsychol 17: $190-195,2010$
27. Gobbi A, Lad D and Karnatzikos G: The effects of repeated intra-articular PRP injections on clinical outcomes of early osteoarthritis of the knee. Knee Surg Sports Traumatol Arthrosc 23: 2170-2177, 2015

28. Filardo G, Kon E, DI Matteo B, DI Marino A, Sessa A, Merli ML and Marcacci M: Leukocyte-poor PRP application for the treatment of knee osteoarthritis. Joints 1: 112-120, 2013.

29. Kelly P, Kahlmeier S, Götschi T, Orsini N, Richards J, Roberts N, Scarborough $\mathrm{P}$ and Foster C: Systematic review and meta-analysis of reduction in all-cause mortality from walking and cycling and shape of dose response relationship. Int J Behav Nutri Phys Act 11: 132, 2014.

30. Babademez MA, Gul F, Muz E, Muderris T and Kale H: Impact of partial and total tonsillectomy on adenoid regrowth. Laryngoscope 127: 753-756, 2017.

31. Spektor Z, Saint-Victor S, Kay DJ and Mandell DL: Risk factors for pediatric post-tonsillectomy hemorrhage. Int J Pediat Otorhinolaryngol 84: 151-155, 2016.

32. Hui D, Kilgore K, Frisbee-Hume S, Park M, Tsao A, Delgado Guay M, Lu C, William W Jr, Pisters K, Eapen G, et al: Dexamethasone for dyspnea in cancer patients: A pilot double-blind, randomized, controlled trial. J Pain Symptom Manage 52: 8-16.e1, 2016

33. Ding L, Zhang H, Mi W, Wang T, He Y, Zhang X, Ma X and $\mathrm{Li} \mathrm{H}$ : Effects of dexmedetomidine on anesthesia recovery period and postoperative cognitive function of patients after robot-assisted laparoscopic radical cystectomy. Int J Clin Exp Med 8: 11388-11395, 2015.

34. Ding L, Zhang H, Mi W, He Y, Zhang X, Ma X and Li H: Effects of dexmedetomidine on recovery period of anesthesia and postoperative cognitive function after robot-assisted laparoscopicradical prostatectomy in the elderly people. Zhong Nan Da Xue Xue Bao Yi Xue Ban 40: 129-135, 2015 (In Chinese).

35. Zhang Z, Li X, Li F and An L: Berberine alleviates postoperative cognitive dysfunction by suppressing neuroinflammation in aged mice. Int Immunopharmacol 38: 426-433, 2016.

36. Yang JY, Lee H, Zhang Y, Lee JU, Park JH and Yun EK: The effects of tonsillectomy education using smartphone text message for mothers and children undergoing tonsillectomy: A randomized controlled trial. Telemed J E Health 22: 921-928, 2016.

37. Kara M, Erdoğan H, Altinişik HB, Aylanç H, Güçlü O and Dereköy FS: Does topical use of autologous serum help to reduce post-tonsillectomy morbidity? A prospective, controlled preliminary study. J Laryngol Otol 130: 662-668, 2016.

38. Drown MB: Integrative review utilizing dexmedetomidine as an anesthetic for monitored anesthesia care and regional anesthesia. Nurs Forum 46: 186-194, 2011.

39. Gerlach AT and Murphy CV: Dexmedetomidine-associated bradycardia progressing to pulseless electrical activity: Case report and review of the literature. Pharmacotherapy 29: 1492, 2009.

40. Srinivas NR: Prostaglandin E1 therapy with alprostadil and risk reduction in early hepatic cellular carcinoma after liver transplantation. Aliment Pharmacol Ther 43: 172-173, 2016.

41. Barton MH, Darden JE, Clifton S and Vandenplas M: Effect of firocoxib on cyclooxygenase 2 , microsomal prostaglandin E2 synthase 1, and cytosolic phospholipase A2 gene expression in equine mononuclear cells. Am J Veter Res 76: 1051-1057, 2015.

42. Antila H, Manner T, Kuurila K, Salanterä S, Kujala R and Aantaa R: Ketoprofen and tramadol for analgesia during early recovery after tonsillectomy in children. Paediatr Anaesth 16 548-553, 2006

43. Yokoyama M: Impact of preventing the postoperative cognitive dysfunction in the aging society. Masui 65: 223, 2016 (In Japanese)

44. Dahmani S, Rouelle D, Gressens P and Mantz J: Effects of dexmedetomidine on hippocampal focal adhesion kinase tyrosine phosphorylation in physiologic and ischemic conditions. Anesthesiology 103: 969-977, 2005.

45. Valentin LS, Pereira VF, Pietrobon RS, Schmidt AP, Oses JP, Portela LV, Souza DO, Vissoci JR, Luz VF, Trintoni LM, et al: Effects of single low dose of dexamethasone before noncardiac and nonneurologic surgery and general anesthesia on postoperative cognitive dysfunction-a phase III double blind, randomized clinical trial. PLoS One 11: e0152308, 2016.

46. Guo Y, Sun L, Zhang J, Li Q, Jiang H and Jiang W: Preventive effects of low-dose dexmedetomidine on postoperative cognitive function and recovery quality in elderly oral cancer patients. Int J Clin Exp Med 8: 16183-16190, 2015. 